Relations industrielles

Industrial Relations

\title{
Michel PRATT : La grève à la United Aircraft. Montréal, Les Presses de l'Université du Québec, 1980, 115 pp.
}

\section{Jacques Gagnon}

Volume 41, numéro 3, 1986

URI : https://id.erudit.org/iderudit/050251ar

DOI : https://doi.org/10.7202/050251ar

Aller au sommaire du numéro

Éditeur(s)

Département des relations industrielles de l'Université Laval

ISSN

0034-379X (imprimé)

1703-8138 (numérique)

Découvrir la revue

Citer ce compte rendu

Gagnon, J. (1986). Compte rendu de [Michel PRATT : La grève à la United Aircraft. Montréal, Les Presses de l'Université du Québec, 1980, 115 pp.] Relations industrielles / Industrial Relations, 41(3), 671-672.

https://doi.org/10.7202/050251ar

Tous droits réservés (C) Département des relations industrielles de l'Université Laval, 1986
Ce document est protégé par la loi sur le droit d'auteur. L’utilisation des services d'Érudit (y compris la reproduction) est assujettie à sa politique d'utilisation que vous pouvez consulter en ligne.

https://apropos.erudit.org/fr/usagers/politique-dutilisation/ 
Néanmoins, la lecture de cette étude n'est pas toujours facile, malgré la clarté de ses conclusions. Les notes au bas des pages (69 en 158 pages) brisent le rythme de lecture. Certaines disgressions théoriques sont quelque peu indigestes. Enfin, quelques concepts utilisés ont mal vieilli, en particulier ceux «d'atomicité» et de «molécularité» des forces en présence. Mais on ne peut que rester impressionné par le volume de travail, la multiplicité des données, la rigueur logique, la franchise et l'honnêteté du professeur Mehling.

La grève de la United Aircraft, par Michel Pratt, Montréal, Les Presses de l'Université du Québec, 1980, 115 pp.

Parce que l'auteur affirme dès le départ son «préjugé défavorable face à la partie patronale», il est assez ironique de constater que son patronyme est le même que celui de l'exUnited Aircraft (Pratt and Whitney). Outre l'introduction et la conclusion, la chronologie et les appendices, son étude comporte quatre chapitres d'inégale longueur: les caractéristiques sociologiques des acteurs (16 pages); le rapport de forces ( 7 pages); la négociation et l'évolution du conflit ( 28 pages); la stratégie des parties ( 24 pages). Mais il faut bien convenir que les deux premiers chapitres sont meilleurs que les deux derniers.

Dans une approche résolument marxiste, ils situent la compagnie américaine et sa filiale canadienne, la centrale syndicale américaine et sa filiale locale, ainsi que le rôle de l'élite d'État canadienne et québécoise. On aurait pu facilement regrouper ces deux chapitres en un seul pour assurer un meilleur équilibre à l'ouvrage. Les troisième et quatrième chapitres sont plus discutables, surtout si on accepte la grille d'analyse marxiste. En effet, on constate que «la masse» fut loin de suivre le mouvement de son «avant-garde» syndicale, bien au contraire. Le nombre de grévistes votants passa de plus de 1500 en décembre 73 et février 74 à moins de 900 à partir de décembre 74. De plus, l'auteur s'étend vraiment trop sur le détail des propositions monétaires successivement soumises et rejetées. Malgré cela, et contrairement à Sauvé et à Mehling, il ne réussit même pas à chiffrer de façon satisfaisante les coûts de la grève pour les syndicats et les syndiqués. Ses données sont trop fragmentaires en ce qui concerne le nombre de grévistes pour chacun des 19 mois de grève (p. 75), la contribution financière totale de la centrale américaine (p. 70) et les autres contributions de grève (dont une soirée de dégustation de cidre et fromage, ainsi que la représentation d'une pièce de théâtre à la Place des Arts)...

Ce qu'on peut retenir du conflit, après lecture et relecture du texte, c'est qu'il fut beaucoup plus long, mais aussi beaucoup plus mouvementé que les deux autres que nous avons revus. Alors que les retours au travail en cours de grève se comptent par quelques centaines à Asbestos - Thetford et à Rouyn-Noranda, ils se chiffrent par plus d'un millier à Longueuil. La violence est endémique pendant une bonne partie de la grève alors qu'elle est sporadique à Asbestos et inexistante à Rouyn-Noranda. Les supports extérieurs sont venus presque exclusivement de la F.T.Q. à laquelle était affilié le syndicat local. Enfin, l'État provincial a agi de façon décisive, par l'intervention personnelle du premier ministre Bourassa. Tout cela suffit-il à faire du conflit de Longueuil «une étape aussi importante dans l'histoire sociale du Québec que la grève de l'amiante de 1949" (p. 2)? Ça reste discutable, dans la mesure où nous avons aussi questionné l'importance du conflit d'Asbestos-Thetford. Une chose est certaine, en tout cas: ni à Asbestos-Thetford, ni à Rouyn-Noranda, ni à Longueuil, la clause de retenue syndicale, jugée essentielle par le syndicat, n'a été inscrite dans le règlement final.

\section{CONCLUSION}

Pour paraphraser le petit catéchisme, nous pouvons dire que nous nous trouvons, au terme de notre recherche, devant trois styles d'étude: une analyse triomphante, celle de Trudeau, une analyse souffrante, celle de Mehling et une analyse militante, celle de Pratt. Bien 
sûr, chacune comporte les défauts de ses qualités. La rhétorique de Trudeau est loin d'être exempte de sophismes. La prudence de Mehling devient parfois trop scrupuleuse. Pratt est souvent trop convaincu pour être convainquant. Mais enfin, s'il fallait conseiller un éventuel rédacteur de monographie de grève, nous lui souhaiterions l'éloquence de Trudeau, l'honnêteté de Mehling, la détermination de Pratt, et la commune sympathie de ces trois auteurs à la cause des travailleurs.

Hormis cette boutade, on peut ajouter au moins trois remarques en guise de conclusion:

$1^{\circ}$ Dans le cas de la grève de l'amiante, la demande de retenue des cotisations syndicales semble avoir été une réclamation moins importante que pour les deux autres grèves étudiées.

$2^{\circ}$ C'est l'étude de Mehling portant sur la grève de Noranda qui, des trois ouvrages, possède le plus grand caractère scientifique dans sa méthode. Si les conclusions n'en sont pas satisfaisantes, c'est parce qu'il était impossible d'arriver à des conclusions plus précises.

$3^{\circ}$ En ce qui concerne le conflit de la United Aircraft, on peut préciser que cette grève était dirigée du côté syndical par un personnage qui, devenu par la suite adjoint au ministre du Travail, a réussi à faire instaurer le précompte syndical obligatoire dans la législation ${ }^{2}$.

Sherbrooke

Jacques GAGNON

2 Ces remarques finales m'ont été obligeamment communiquées par le Comité de lecture de la revue et je l'en remercie. Jacques Gagnon

\title{
LA MOBILISATION DES RESSOURCES HUMAINES TENDANCES ET IMPACT
}

\begin{abstract}
Préface, Laurent BÉLANGER - Introduction, Michel AUDET, Laurent BÉLANGER, Jean BOIVIN, Esther DEOM, Jacques MERCIER - PARTIE 1: TENDANCES RECENTES EN GESTION DES RESSOURCES HUMAINES - Emergence d'une réalité nouvelle en relations industrielles, Jean BOIVIN - La mobilisation des ressources humaines: orientations récentes, Viateur LAROUCHE - La stratégie des ressources humaines à la Compagnie Abitibi-Price Inc., Jean-Claude CASAVANT - La communication directe chez Cascades Inc., Alain LEMAIRE - Le projet d'entreprise de Culinar Inc., Roger NERON - Les limites des nouvelles approches en gestion des ressources humaines, Lysette BOUCHER - L'entreprise du $3^{\mathrm{e}}$ type, Hervé SERIEYX - Les travailleurs seront-ils du $3^{\mathrm{e}}$ type?, Thierry WILS - Le conflit: la gestion au banc des accusés, Yves DULUDE - Commentaire, Pierre LAMARCHE - L'expérience de la Compagnie Gaz Métropolitain Inc., Serge LALANDE - PARTIE II: IMPACT SUR LES ORGANISATIONS SYNDICALES ET LA NÉGOCIATION COLLECTIVE - Ressources humaines et défis du syndicalisme, Gérard DOCQUIER - Le syndicalisme et le nouveau travail, Jean FRANCOEUR - La nouvelle gestion des ressources humaines: mythe ou réalité?, Jean-Paul HETU - Les préalables à une ré-orientation des relations du travail du Québec, Louis LABERGE - L'adaptation du syndicalisme: un phénomène de continuité, Gérald LAROSE - L'avenir de la négociation collective, Thomas A. KOCHAN.
\end{abstract}

ISBN 2-7637-7107-7

1 volume, 1986,199 pages $-\$ 17.00$

Les Presses de l'Université Laval

Cité universitaire

C.P. 2447, Québec, P.Q., Canada

G1K 7R4 